\title{
Microperimetry and optical coherence tomography imaging in the fellow eye of patients with unilateral focal ischaemic glaucoma
}

\author{
Imran H. Yusuf ${ }^{1}$ · Jasleen K. Jolly $\mathbb{B}^{1}{ }^{1}$. Gokulan Ratnarajan ${ }^{1} \cdot$ John F. Salmon ${ }^{1}$ \\ Received: 14 November 2017 / Revised: 1 March 2018 / Accepted: 2 March 2018 / Published online: 30 April 2018 \\ (c) The Royal College of Ophthalmologists 2018
}

\begin{abstract}
Purpose To determine whether microperimetry or optical coherence tomography (OCT) imaging can detect early disease in the fellow eye of patients with unilateral focal ischaemic glaucoma.

Methods Thirty-seven consecutive adult patients with unilateral focal ischaemic glaucoma with a unilateral split-fixation visual field defect on standard automated perimetry (SAP) with normal SAP in the fellow eye were selected. All patients underwent microperimetry (MAIA, CenterVue, Italy) of the central 10 degrees and OCT imaging (Spectralis, Heidelberg Engineering, Germany) of the retinal nerve fibre layer of both eyes. The main outcome measures were reduced retinal sensitivity on microperimetry and/or retinal nerve fibre layer thinning on OCT imaging of the fellow eye.

Results Thirty fellow eyes had abnormal global thresholds on microperimetry, and 20 had abnormal OCT imaging studies. Kappa agreement between tests in fellow eyes was poor $(p=0.2546)$. Fixation was significantly poorer in fellow eyes on microperimetry when compared to eyes with glaucoma $(p<0.003)$. In the fellow eyes that were abnormal, microperimetry identified reduced retinal sensitivity at fixation.

Conclusion Microperimetry detects reduced retinal sensitivity close to fixation and OCT detects focal thinning of the retinal nerve fibre layer in the fellow eye of most patients with presumed unilateral focal ischaemic glaucoma. Further studies are required to correlate specific optic disc features on OCT imaging with microperimetry in the fellow eye of this patient group.
\end{abstract}

\section{Introduction}

Glaucoma is the most common cause of irreversible blindness worldwide, characterised by permanent loss of retinal ganglion cells [1]. Early deficits in the peripheral or paracentral visual field are common in many forms of glaucomatous optic neuropathy. Focal ischaemic glaucoma is a disease subtype characterised by focal notching of the neuroretinal rim with a congruous visual field deficit, which threatens fixation early in the clinical course ("split-fixation" visual field defect) $[2,3]$. Female gender, earlier age

Electronic supplementary material The online version of this article (https://doi.org/10.1038/s41433-018-0094-3) contains supplementary material, which is available to authorized users.

John F. Salmon

john.salmon@ouh.nhs.uk

1 The Oxford Eye Hospital, West Wing, John Radcliffe Hospital, John Radcliffe Hospital, Headley Way, Headington, Oxford OX3 9DU, UK of onset, recurrent optic disc haemorrhages and primary vascular dysregulation syndromes (nocturnal hypotension, vasospasm, migraine and Raynaud's phenomenon) are associated with the focal ischaemic glaucoma subtype [2-7].

Focal ischaemic glaucoma results in loss of macular sensitivity at or near the point of fixation [8]. Early identification of glaucoma in the fellow eyes of this patient group is critical to reduce the risk of irreversible, bilateral central visual field defects, which may threaten reading ability and legal visual thresholds for driving. Early visual field deficits have a negative impact on quality-of-life measures [9]. Defects in the neuroretinal rim identified by clinical appearances or optic nerve imaging may exist prior to visual field loss detected by standard automated perimetry (SAP) $[10,11]$. It follows that more sensitive functional perimetric tools must be identified. Microperimetry (fundus driven perimetry) is a novel diagnostic tool that can evaluate macular sensitivity at specific retinal locations. Microperimetry has been used as an outcome measure in some clinical studies of glaucoma, although it is limited to the evaluation of macular rather than peripheral retinal 
sensitivity $[12,13]$. The utility of microperimetry to detect early focal ischaemic glaucoma, where visual field loss often starts near fixation, has not been evaluated.

This study sought to evaluate the utility of microperimetry and optical coherence tomography (OCT) imaging of the peripapillary retinal nerve fibre layer (RNFL) to detect early abnormalities in the fellow eye of patients with presumed unilateral focal ischaemic glaucoma who achieved a full visual field on SAP in the fellow eye. Additionally, we sought to determine whether there was agreement between microperimetry assessment and OCT imaging if abnormalities were identified in both tests in fellow eyes.

\section{Methods}

\section{Patient selection}

Patients were recruited prospectively from the glaucoma clinic in a single-centre tertiary referral ophthalmology unit (Oxford Eye Hospital, John Radcliffe Hospital, Oxford, UK). Patients who fulfilled the inclusion criteria and agreed to participate were selected consecutively between February 2014 and June 2015: 37 patients were identified for inclusion in the study. Each participant provided informed consent prior to participation in accordance with the Declaration of Helsinki (Local approval number: 4281).

\section{Inclusion criteria}

Adult patients (age $>18$ years); unilateral primary focal ischaemic glaucomatous optic neuropathy-defined as focal neuroretinal rim loss on clinical examination confirmed objectively by OCT imaging of the peripapillary retinal nerve fibre layer; and congruous unilateral visual field loss close to fixation in the affected eye detected on SAP (Humphrey 24-2, SITA standard). The scotoma was defined as: three contiguous points $(p<0.5)$ within 5 degrees of fixation with least one point identified as $p<$ $0.05 \%$ on pattern deviation and outside normal limits using the glaucoma hemifield test.

Normal SAP was found in all cases in the fellow eye (Humphrey 24-2, SITA standard), defined as: absence of three adjacent points $<1 \%$ on pattern deviation, no cluster of three points involving a point $p<0.5 \%$ on pattern deviation, and normal glaucoma hemifield test result. OCT imaging was not used in the fellow eyes to determine eligibility for the study.

\section{Exclusion criteria}

Best-corrected visual acuity of LogMAR 0.6 or worse in either eye; myopia of $>-8.00 \mathrm{DS}$ or hyperopia of
$>+6.00 \mathrm{DS}$; inability to perform perimetry for any reason; macular pathology in healthy, fellow eye detected clinically or on OCT imaging of the macula; non-glaucomatous optic nerve pathology; significant lens or posterior capsular opacity in the fellow eye; ocular history of significant additional pathology (diabetic retinopathy, trauma, intraocular inflammation); clinically significant ptosis that interfered with SAP testing; previous intraocular surgery other than cataract surgery (patients with previous trabeculectomy were excluded); and secondary glaucoma (pseudoexfoliation, pigmentary glaucoma, neovascular or inflammatory glaucoma).

\section{Study protocol}

A detailed ophthalmic and medical history was taken from each patient. Ophthalmic examination was performed including slit lamp biomicroscopy, Goldmann applanation tonometry, gonioscopy and fundoscopy assessment. Bestcorrected visual acuity was measured using LogMAR acuity charts. At least two reliable, reproducible SAP examinations were performed on different days on both eyes (Humprey 24-2 SITA standard; Humphrey field analyser, model 750, Zeiss-Humphrey Systems, Dublin, CA, USA). Humphrey visual field (HVF) analysis was considered of sufficient quality if fixation losses were $<20 \%$ and false positives and false negatives $<10 \%$. The most reliable SAP test was included in, and determined eligibility for, the study. Digital optic disc photographs and OCT imaging (Spectralis, Heidelberg Engineering, Heidelberg, Germany) of the peripapillary RNFL and macula were undertaken on both eyes of each patient. Intraocular pressure at presentation and use of topical antihypertensive therapies were documented for each patient, although neither parameter prevented participation in the study. All patients were selected by a single, experienced glaucoma specialist (JFS) with specific reference to the exclusion and inclusion criteria. Thirty-seven patients (74 eyes) were analysed.

Table 1 describes the baseline clinical characteristics of the study subjects.

\section{Microperimetry}

Each subject underwent dark adaptation for at least $15 \mathrm{~min}$ prior to examination. Microperimetry examination was conducted with the MAIA Microperimeter (CentreVue, Padova, Italy). The scanning laser ophthalmoscopic image of the macula was inspected prior to the start of the study to exclude structural pathology. The MAIA utilises a Goldmann size III stimulus $(200 \mathrm{~ms})$ projected onto a white background with illumination of $1.27 \mathrm{~cd} / \mathrm{m}^{2}$. A full threshold strategy using 4-2-1 staircase method was used to test 
Table 1 Clinical characteristics of the study subjects

\begin{tabular}{|c|c|c|}
\hline & Glaucoma eye & Study eye \\
\hline Age (years) & 70 (48 to 82$)$ & \\
\hline \multicolumn{3}{|l|}{ Sex } \\
\hline Male & 14 (mean age-70.9) & \\
\hline Female & 23 (mean age-69.1) & \\
\hline Visual acuity (LogMAR) & $-0.07(-0.20$ to +0.30$)$ & $-0.08(-0.20$ to +0.10$)$ \\
\hline Central corneal thickness (microns) & $538(450$ to 616$)$ & 539 (451 to 632$)$ \\
\hline IOP at presentation $(\mathrm{mmHg})(n=30)$ & 19.1 (13 to 28$)$ & $18.4(13$ to 28$)$ \\
\hline Number of topical glaucoma agents & $1.64(0$ to 3$)$ & $1.43(0$ to 3$)$ \\
\hline \multicolumn{3}{|l|}{ Humphrey visual field (24-2) } \\
\hline Mean deviation & $-4.65(-14.02$ to -0.31$)$ & $-0.59(-2.16$ to 1.99$)$ \\
\hline Pattern standard deviation & 7.86 (3.42 to 13.54$)$ & 1.84 (1.16 to 3.08$)$ \\
\hline Microperimetry average threshold (dB) (mean sensitivity) & $18.61(7.5$ to 25.9$)$ & 26.41 (23.4 to 29.8$)$ \\
\hline Fixation area covering $95 \%$ of fixation points (degrees) & $1.6(0.3$ to 4.7$)$ & $3.0(0.0$ to 14.9$)$ \\
\hline \multicolumn{3}{|l|}{ OCT imaging of RNFL } \\
\hline Global index & 71.5 (49 to 99$)$ & 84.1 (66 to 110$)$ \\
\hline Superior & 93.9 (57 to 147$)$ & 99.8 (65 to 127$)$ \\
\hline Inferior & 75.0 (39 to 134$)$ & 102.5 (53 to 145$)$ \\
\hline Nasal & 66.1 (37 to 99$)$ & 70.0 (51 to 97$)$ \\
\hline Temporal & $51.2(29$ to 64$)$ & $64.0(41$ to 88$)$ \\
\hline Migraine (\%) & $16.2 \%$ (6 patients) & \\
\hline Raynaud's phenomenon (\%) & $24.3 \%$ (9 patients) & \\
\hline
\end{tabular}

Mean values are presented with range of values in parentheses

37 points within the central 10 degrees of the visual field (Fig. 1a-c). This test took around 5 min per eye. The eye with documented glaucoma was examined first, followed by the fellow, study eye. The MAIA software automatically tracks eye movement and records all fixation points throughout the examination. The time between clinical evaluation and microperimetry examination was $<4$ weeks for each patient.

\section{Outcome measures}

The main outcome measures for the study were abnormalities detected in the fellow eyes: thinning of the peripapillary RNFL on OCT imaging, reduction in macular sensitivity on microperimetry assessment, and abnormal fixation behaviour on microperimetry assessment.

\section{Statistical analysis}

Normality was determined using the Shapiro-Wilk method. Paired $t$-tests or Wilcoxon signed-rank tests were used to compare the findings of two eyes. Spearman rank correlation was used to assess the association between microperimetry and OCT. This was explored further with Kappa agreement.
In order to determine the utility of microperimetry in the early detection of glaucoma in the fellow eyes, two assessments were undertaken on each fellow eye. First, the average of the lowest five threshold points on microperimetry was compared to eyes designated as red (outside of normal limits in comparison to a normative database) on OCT imaging and those designated as green (within normal limits in comparison to normative database). This designation is made by Heidelberg Spectralis imaging software based on comparisons to a normative database. Fixation area for $95 \%$ of the fixation plots was compared between the eyes using Wilcoxon signed-rank test.

In order to determine the level of agreement between microperimetry and OCT imaging of the RNFL, the findings of each test in fellow eyes were tabulated (Table 2). In order to compare the results of superior and inferior hemifields on microperimetry, we selected data as illustrated in Supplement 3: points lying along the horizontal raphe were excluded, and three separate rings (inner, middle and outer) were analysed separately. Fifteen points from the superior hemifield and 15 points from the inferior hemifield were included in the analysis.

All statistics were conducted using StatsDirect (StatsDirect Ltd, Cheshire, UK). The retinal sensitivity at each point was summated and created into a shapefile using 
a

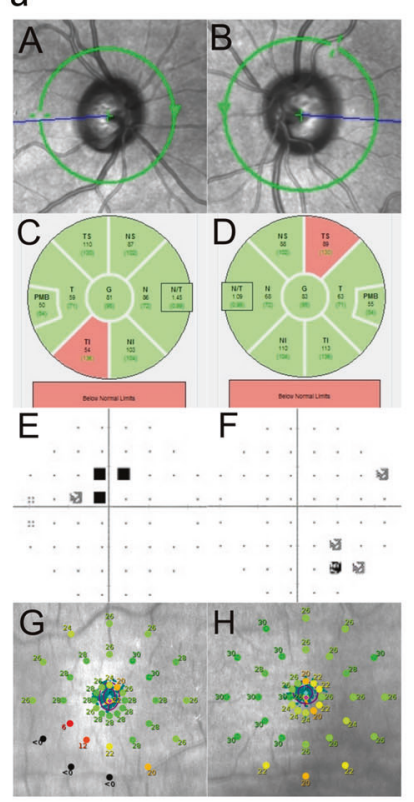

b

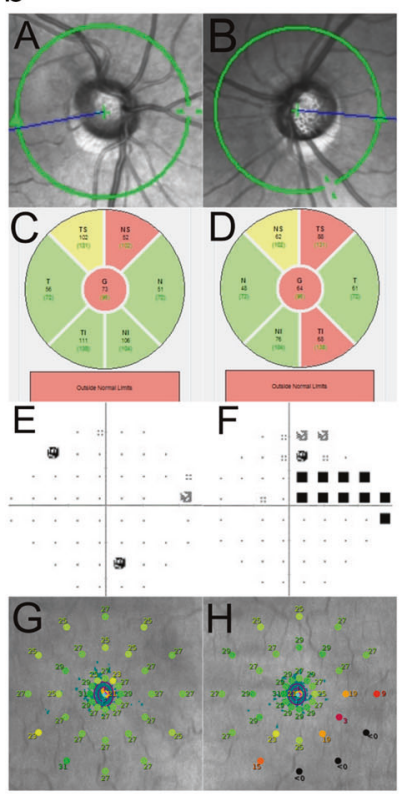

C

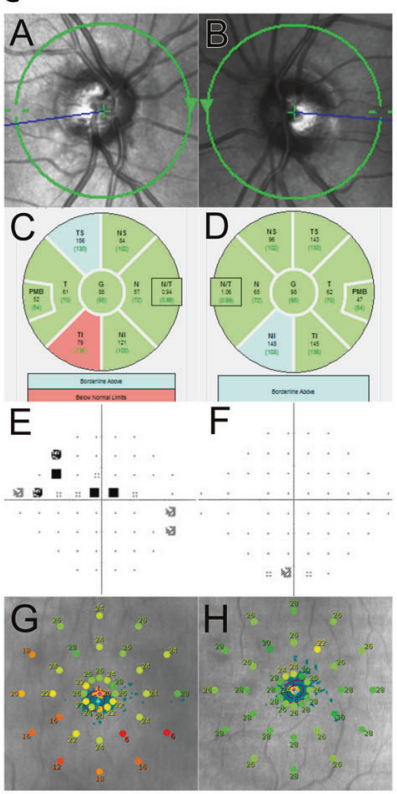

Fig. 1 a Study subject with abnormal OCT imaging and microperimetry plot in fellow eye. (a, b) Optic disc scanning laser ophthalmoscopic images demonstrate an inferior notch in the right optic disc with focal ischaemic glaucoma and a healthy appearing optic disc on the left. (c, d) OCT of peripapillary retinal nerve fibre layer demonstrating thinning of the right infero-temporal and left superotemporal RNFL. (e, f) Standard automated perimetry (24-2, SITA standard) pattern standard deviation plots of right and left eyes demonstrating a split-fixation visual field defect in the right eye (supero-nasal). The left PSD plot shows non-specific visual field changes in the infero-nasal quadrant. ( $\mathrm{g}, \mathrm{h}$ ) Microperimetry plots demonstrate reduced retinal sensitivity in the infero-temporal macula close to fixation in the right eye and reduced retinal sensitivity in the inner supero-temporal ring of the left eye. b Study subject with abnormal OCT imaging and microperimetry in the fellow eye. $(a, b)$ Optic disc SLO images demonstrate a left inferior notch with peripapillary atrophy. The right optic disc demonstrates peripapillary atrophy with mild tilting of the optic disc but no clear notch. (c, d) OCT scan of peripapillary RNFL demonstrating thinning of the right supero-nasal
RNFL and left supero-temoral and infero-temporal RNFL. (e, f) Standard automated perimetry pattern standard deviation plots demonstrate a superior visual field defect in the left eye, splitting fixation. The right eye demonstrates non-specific changes with normal glaucoma hemifield test. ( $g$, h) Microperimetry plots demonstrate reduced retinal sensitivity in the infero-temporal macula close to fixation in the left eye. The right eye demonstrates reduced retinal sensitivity in the superior macula when compared to the inferior macula. c A study subject with normal OCT imaging and abnormal microperimetry in fellow eye. $(a, b)$ SLO images demonstrate a right inferior notch and healthy appearing left optic disc. (c, d) OCT demonstrates thinning of the right infero-temporal RNFL, the left RNFL is normal. (e, f) HVF PSD plots demonstrate a scotoma superiorly in the right eye close to fixation, most prominent in the supero-nasal sector. The left visual field is full. $(\mathrm{g}, \mathrm{h})$ Microperimetry plots demonstrate a reduction in retinal sensitivity in the right inferior macula. The left microperimetry plot demonstrates reduced retinal sensitivity in the superior macula within the inner ring (with abnormal global index reported by the MAIA software)
Table 2 Agreement between microperimetry and OCT imaging in the study eyes

\begin{tabular}{lll}
\hline & Microperimetry normal & Microperimetry abnormal \\
\hline OCT normal & 4 & 13 \\
OCT abnormal & 3 & 17 \\
\hline
\end{tabular}

Values indicate the number of patients who demonstrate a normal or abnormal OCT imaging or microperimetry test results in the study eye. Normal or abnormal tests results were defined by age-adjusted automated analyses within the Heidelberg Spectralis and MAIA Microperimeter, respectively

FME $^{\circledR}$ (Safe Software Inc, Canada). This was converted to a colour-coded "heat map" of retinal sensitivity across the different points using QGIS (version 2.6, open source Geographic Information Systems software).

\section{Results}

\section{Microperimetry thresholds and fixation behaviour between glaucoma and fellow eyes}

Average microperimetry threshold in the glaucoma eyes was $18.6 \pm 4.4 \mathrm{~dB}$ versus $26.4 \pm 1.4 \mathrm{~dB}$ in the fellow eyes $(p<0.05)$. Average microperimetry thresholds were compared between the eyes with focal ischaemic glaucoma and fellow eyes. Shapiro-Wilk testing demonstrated that the data for the glaucoma eyes were not normally distributed, although fellow eye data were. Wilcoxon signed-rank test was used to compare the microperimetry average thresholds between the two eyes that were different, as expected ( $p<$ 0.0001; Fig. 2a). The mean fixation area was 1.63 degrees squared for glaucoma eyes and 3.0 degrees squared for 

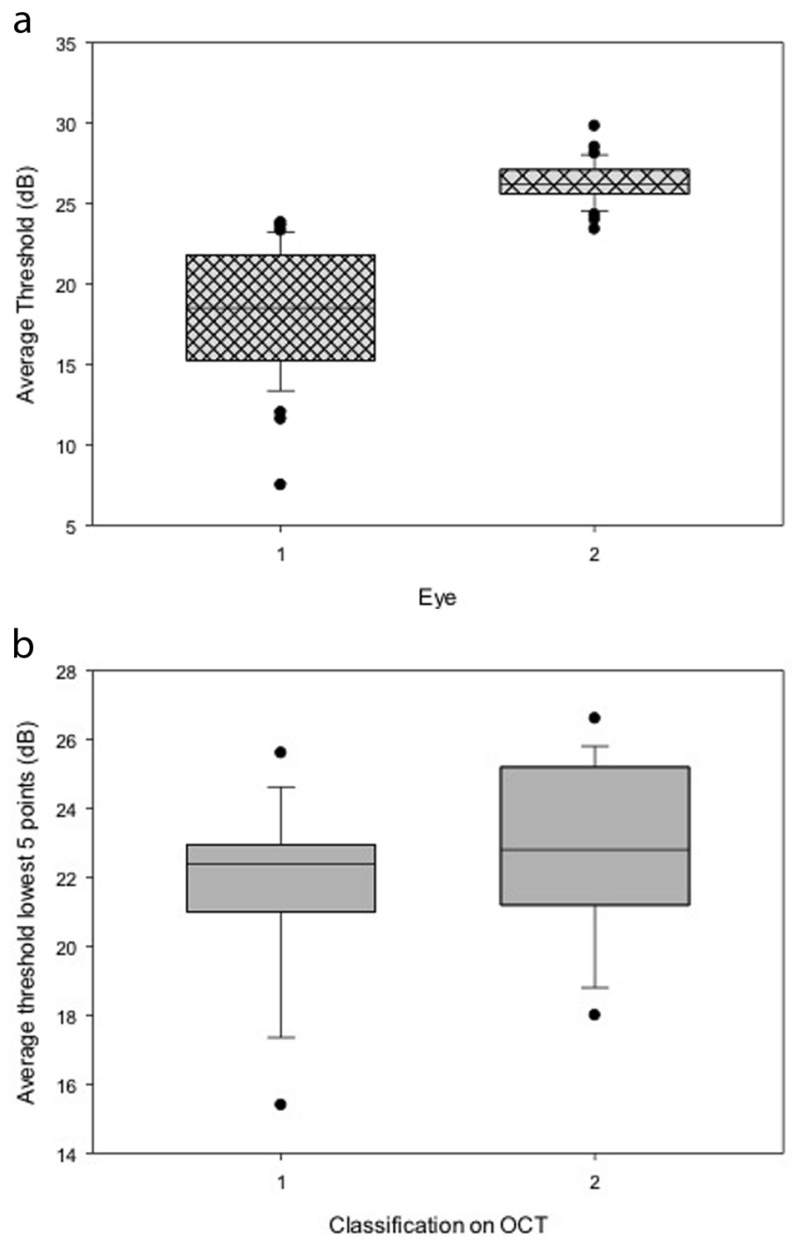

Fig. 2 a Comparison of average microperimetry thresholds in focal ischaemic glaucoma eyes (1) and fellow study eyes (2). A clear reduction in threshold sensitivity in the eyes with focal ischemic glaucoma is demonstrated $(p<0.0001)$ (dB decibels). b Microperimetry thresholds (average) in the fellow eyes when compared between OCT classifications. (1) Patients with fellow eye classed as RED (significant thinning) on OCT global index of RNFL thickness when compared to age-matched controls. (2) Patients with fellow eye classed as GREEN (no thinning) on OCT global index of RNFL thickness. This demonstrates no difference between the two groups: microperimetry does not detect differences between patients with structurally normal and structurally abnormal peripapillary RNFL thickness on OCT imaging. $\mathrm{dB}$ decibels

fellow eyes (Supplement 1). Using Wilcoxon signed-rank test, this difference was found to be significant $(p<0.003)$.

\section{OCT imaging of RNFL between glaucoma and fellow eyes}

The average RNFL thickness was $71.7 \pm 11.6 \mu \mathrm{m}$ in the glaucoma eyes versus $85.6 \pm 11.3 \mu \mathrm{m}$ in fellow eyes $(p<$ $0.05)$. The average RNFL thickness measurements on OCT imaging (global value) were compared between eyes with focal ischaemic glaucoma and fellow eyes. Shapiro-Wilk demonstrated normality of both data sets. Paired $t$-test demonstrated that the two eyes significantly differed from each other $(p<0.0001)$.

\section{Microperimetry and OCT imaging in the glaucoma and fellow eyes: global indices}

There was no difference between eyes with glaucoma and the fellow eyes when global OCT measurements and global microperimetry thresholds were analysed (Spearman's rank correlation).

\section{Microperimetry sensitivity according to OCT RNFL thickness designation}

There was no significant difference between the microperimetry average of the lowest five values in patient's fellow eyes when those fellow eyes designated OCT imaging as "outside of normal limits" were compared with those designated as "within normal limits" (Fig. 2b; Table 2).

\section{Microperimetry and OCT imaging of RNFL in the fellow eyes}

Out of the 37 fellow eyes, 30 had abnormal global retinal sensitivity thresholds on microperimetry, and 20 had abnormal OCT imaging studies (Table 2 and Fig. 1). When these tests were combined, only four fellow eyes had normal OCT imaging results and normal retinal sensitivity on microperimetry. Kappa agreement for microperimetry and OCT for fellow eyes was $0.089231(\mathrm{SE}=0.135165)$. The $95 \%$ confidence interval for kappa $=-0.175688$ to 0.35415 $(p=0.2546)$.

\section{Microperimetry: pattern of loss of retinal sensitivity in the glaucoma and fellow eyes}

Two-way analysis of variance testing with Bonferroni correction for multiple testing showed a significant difference between the inner ring and the other rings on microperimetry assessment for both glaucoma eyes and fellow eyes $(p<0.005)$. In the fellow eyes, the difference fell within test-retest limits but not in the eyes with glaucoma (Supplement 2).

An average of retinal sensitivity measurements in the glaucoma and fellow eyes as determined by microperimetry at each retinal locus is presented in Fig. 3. This demonstrates reduced retinal sensitivity in the inferior macula in patients with focal ischaemic glaucoma. In the fellow eyes, reduced retinal sensitivity is demonstrated at fixation and inferiorly within the inner ring ( 2 degrees of eccentricity) and outer ring with preservation of retinal sensitivity in the middle ring. 

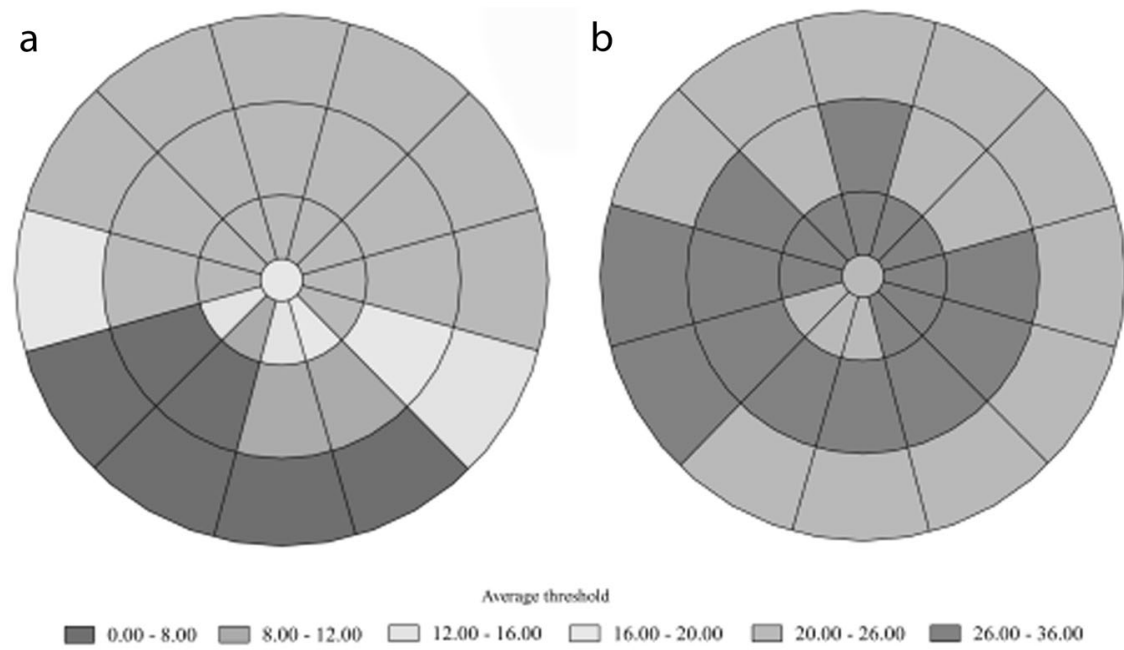

Fig. 3 Summation of retinal sensitivity as determined by microperimetry in $\mathbf{a}$ eyes with focal ischaemic glaucoma and $\mathbf{b}$ fellow eyes. The plots represent retinal sensitivity rather than visual field abnormalities. The inferior retina appears to have reduced sensitivity in the eyes with glaucoma. The fellow eyes have reduced retinal

\section{Discussion}

In this study, we have identified that most patients with presumed unilateral focal ischaemic glaucoma with a normal SAP examination in the fellow eye have abnormalities on OCT imaging of the RNFL (54\%) or reduced retinal sensitivity on microperimetry $(81 \%)$ in the fellow eye. In all, $89 \%$ of patients had abnormalities on either microperimetry or OCT imaging and only 4 patients out of the 37 with presumed unilateral disease had a normal examination with both OCT imaging and microperimetry examination in the fellow eye.

Microperimetry is an ideal functional tool to detect subtle visual field deficits in the fellow eyes in this patient group where it is assumed that scotomas are likely to develop close to fixation. This subjective test is becoming established as a reproducible outcome measure in clinical trials of macular disease in the evaluation of thresholds of retinal sensitivity and fixation behaviour [14]. In the context of clinical glaucoma studies, microperimetry is most often used as a tool to evaluate the functional characteristics of the central 10 degrees around the fovea but cannot currently evaluate visual field deficits in the peripheral retina $[13,15-$ 17]. The real-time eye tracking and adjustments made during testing in theory increase the reliability of the measures of threshold due to the consistency of the visual space being tested throughout the examination, though this has not been independently verified. However, MAIA have developed a new microperimeter (Compass), which may be used in further clinical studies to directly compare $24-2$ HVF testing with a microperimetry assessment over the sensitivity in the centre of the fovea and inferiorly close to fixation. The difference in retinal sensitivity between superior and inferior retina increases with eccentricity, such that the outer ring is most abnormal in comparison to the inner and middle rings (as demonstrated in Supplementary Figure 2)

same field. This would allow a calculation of the sensitivities and specificities of each test in this patient group.

In patients in this study where fixation is threatened in the eye with glaucoma, it is surprising to detect statistically significant poorer fixation in the fellow eye. The reasons for this finding are unclear, although it may be possible that eyes with glaucoma have adapted to maintain steady fixation with a small, central visual field defect. Additionally, the test-retest variability of microperimetry has been defined in one study and found to be superior in the control eyes when compared with eyes with fixation-threatening glaucoma [18]. Fixation instability on microperimetry has been suggested as an early marker of glaucoma [19].

The finding that $81 \%$ of patients had an abnormality on microperimetry in the presumed normal fellow eye was unexpected given that the SAP results were normal. An abnormality was defined as an abnormal global macular integrity index. This index is determined by the MAIA software's internal database, which compares the responses of patients with those obtained from normal age-matched controls. The software produces a histogram defining the normal range of thresholds for the points tested within the central area. Each point is compared to this database and colour coded as to the threshold and the probability of it being outside of the normal range (Fig. 1). Any points with colour coding yellow or warmer were classified as abnormal as this puts it firmly outside the normal range according to threshold. When a cluster of abnormal points were seen, the field plot can be considered abnormal. In order to limit bias, we performed microperimetry assessment on the fellow eye following examination of the eye with focal ischaemic glaucoma 
to allow any learning effect to advantage microperimetry performance in the fellow eye. It is known that threshold values are often lower on microperimetry when compared to SAP: mean macular sensitivity was found to be significantly lower on microperimetry when compared to SAP on the same test subjects with advanced primary open angle glaucoma [13]. This is likely to reflect differing test sensitivities.

For established visual field deficits, clinical studies have identified a significant correlation between 10-2 SAP and microperimetry in affected quadrants in glaucoma. However, a previous study has shown that $21 \%$ of the normal quadrants on SAP had an abnormal microperimetry result, and of these, $75 \%$ had abnormal structural imaging on OCT imaging, although the proportion of patients with normal or abnormal quadrants on OCT imaging who had abnormal microperimetry was not reported [20]. We have identified in this study that abnormalities on microperimetry were more common than abnormalities on OCT imaging in the fellow eye of patients with focal ischaemic glaucoma using global indices. This may reflect the limitations of a subjective test or may represent reduced sensitivity in macular regions corresponding to focal dysfunctional retinal ganglion cells prior to retinal ganglion cell death and RNFL thinning. Our findings of microperimetry abnormalities in patients with normal SAP examination is consistent with findings in a previous study of glaucoma patients with unselected glaucoma subtypes [20]. In patients with very advanced glaucoma, microperimetry has been explored as a tool to determine progression [21]. Microperimetry, without automated fundus tracking, was able to detect areas of reduced retinal sensitivity in the context of focal RNFL defects detected on Argon blue SLO imaging, detected in only 14 of the 25 eyes with standard perimetry [22].

Microperimetry also permits an analysis of the location of reduced retinal sensitivity within the macula in patients with focal ischaemic glaucoma. In this study, microperimetry examination of patients with focal ischaemic glaucoma identified infero-temporal paracentral reduction in macular sensitivity more prominent at 6 and 10 degrees eccentricity when responses from all 37 subjects were summated. Responses at 2 degrees were relatively spared. Other studies have explored the correlation between ganglion cell inner plexiform thickness and Cirrus OCT finding correlation in all visual field/retinal sectors, most prominently inferiorly and temporally [23]. Glaucomatous structural and functional changes may be most prominent in this retinal region, as demonstrated in other studies [17]. In the fellow eyes, there appeared to be reduced sensitivity inferotemporally at 2 degrees and at fixation with preservation of more eccentric responses. The reasons for this pattern of loss of retinal sensitivity are unclear.

RNFL indices on OCT imaging correlated in one study with both microperimetry and SAP [13]. We did not find a structure-functional correlation when examining the lowest microperimetry thresholds in patients with normal and abnormal OCT classifications in the fellow eyes. This may be in part because the depth of RNFL thinning covers a wide range of RNFL thicknesses within a single classification (i.e. subtle and severe thinning may both be classified as outside normal limits on OCT imaging). However, the absence of correlation between microperimetry and OCT imaging in the fellow eyes suggests a poor structure-function correlation in early disease. Therefore, as with SAP, abnormalities on microperimetry may be considered non-specific and not necessarily congruous to identified structural defects on OCT imaging.

OCT imaging of the peripapillary RNFL in this study identified that many fellow eyes (20 out of the 37 eyes) demonstrate an abnormally thin RNFL in at least one quadrant when compared to normative databases of healthy, age-matched controls that is unsurprising given the results of microperimetry. A follow-up study to determine which of these patients in time develop visual field loss detectable on either microperimetry or later with SAP would be of interest. In particular, it would be useful to determine whether OCT imaging or retinal sensitivity on microperimetry is more predictive and whether those patients with abnormal test results with both modalities are more likely to develop glaucoma detectable on SAP. One of the limitations of this study was the inability to define the specificity of both microperimetry and OCT imaging in the context of focal ischaemic glaucoma; further studies are required to determine this. New microperimeters that allow a direct comparison with 24-2 HVF testing with similar test grids are likely to make such a study possible.

In conclusion, focal ischaemic glaucoma is characterised by early visual field deficits threatening fixation [2, 7, 24]. In patients with unilateral disease, early detection of disease in the fellow eye and the subsequent treatment is critical to prevent bilateral central scotomas, which may threaten reading or driving vision [9]. SAP is often the primary diagnostic tool used to detect early disease in the fellow eye. This study demonstrates that microperimetry and OCT imaging of the RNFL may detect early disease in the fellow eyes of patients with presumed unilateral focal ischaemic glaucoma who have a normal SAP examination in the fellow eye. These tests may serve an important clinical utility for reducing the risk of bilateral visual loss in this patient group.

\section{Summary}

\section{What was known before}

- Focal ischaemic glaucoma is associated with visual loss threatening fixation early in the clinical course. 
- Early detection of disease in fellow eyes of patients with presumed unilateral glaucoma is important to prevent bilateral scotomas at fixation.

- Microperimetry is a form of fundus tracking perimetry that has not been evaluated in this patient group.

\section{What this study adds}

- Microperimetry and optical coherence tomography imaging of the retinal nerve fibre layer detect abnormalities in most fellow eyes of patients with presumed unilateral focal ischaemic glaucoma.

- Microperimetry appears to have a greater sensitivity than OCT imaging.

- Microperimetry, where available, should be considered for the early detection of glaucoma in the fellow eyes of this patient group.

Acknowledgements $\mathrm{FME}^{\circledR}$ license provided on a non-profit research grant to JKJ.

\section{Compliance with ethical standards}

Conflict of interest The authors declare that they have no conflict of interest.

\section{References}

1. Tham YC, Li X, Wong TY, Quigley HA, Aung T, Cheng CY. Global prevalence of glaucoma and projections of glaucoma burden through 2040: a systematic review and meta-analysis. Ophthalmology. 2014;121:2081-90.

2. Nicolela MT, Drance SM. Various glaucomatous optic nerve appearances: clinical correlations. Ophthalmology. 1996;103: 640-9.

3. Nicolela MT, Walman BE, Buckley AR, Drance SM. Various glaucomatous optic nerve appearances. A color Doppler imaging study of retrobulbar circulation. Ophthalmology. 1996;103:1670-9.

4. Bechetoille A, Bresson-Dumont H. Diurnal and nocturnal blood pressure drops in patients with focal ischemic glaucoma. Graefes Arch Clin Exp Ophthalmol. 1994;232:675-9.

5. Yamazaki Y, Hayamizu F, Miyamoto S, Nakagami T, Tanaka C, Inui S. Optic disc findings in normal tension glaucoma. Jpn J Ophthalmol. 1997;41:260-7.

6. Broadway DC, Drance SM. Glaucoma and vasospasm. Br J Ophthalmol. 1998;82:862-70.

7. Takada N, Omodaka K, Kikawa T, Takagi A, Matsumoto A, Yokoyama Y, et al. OCT-based quantification and classification of optic disc structure in glaucoma patients. PLoS ONE. 2016;11: $\mathrm{e} 0160226$.

8. Nakazawa T, Shimura M, Ryu M, Himori N, Nitta F, Omodaka K, et al. Progression of visual field defects in eyes with different optic disc appearances in patients with normal tension glaucoma. J Glaucoma. 2012;21:426-30.
9. McKean-Cowdin R, Varma R, Wu J, Hays RD, Azen SP. Severity of visual field loss and health-related quality of life. Am J Ophthalmol. 2007;143:1013-23.

10. Klamann MK, Grunert A, Maier AK, Gonnermann J, Joussen AM, Huber KK. Comparison of functional and morphological diagnostics in glaucoma patients and healthy subjects. Ophthalmic Res. 2013;49:192-8.

11. Hood DC, Slobodnick A, Raza AS, de Moraes CG, Teng CC, Ritch R. Early glaucoma involves both deep local, and shallow widespread, retinal nerve fiber damage of the macular region. Invest Ophthalmol Vis Sci. 2014;55:632-49.

12. Rao HL, Januwada M, Hussain RS, Pillutla LN, Begum VU, Chaitanya A, et al. Comparing the structure-function relationship at the macula with standard automated perimetry and microperimetry. Invest Ophthalmol Vis Sci. 2015;56:8063-8.

13. Kulkarni SV, Coupland SG, Stitt DM, Hamilton J, Brownstein JJ, Damji KF. Efficacy of SLO-Microperimetry and Humphrey for evaluating macular sensitivity changes in advanced glaucoma. Can J Ophthalmol. 2013;48:406-12.

14. Rao HL, Hussain RS, Januwada M, Pillutla LN, Begum VU, Chaitanya A, et al. Structural and functional assessment of macula to diagnose glaucoma. Eye. 2017;31:593-600.

15. Ozturk F, Yavas GF, Kusbeci T, Ermis SS. A comparison among Humphrey field analyzer, microperimetry, and Heidelberg retina tomograph in the evaluation of macula in primary open angle glaucoma. J Glaucoma. 2008;17:118-21.

16. Shi Y, Liu M, Wang X, Zhang C, Huang P. Fixation behavior in primary open angle glaucoma at early and moderate stage assessed by the MicroPerimeter MP-1. J Glaucoma. 2013;22:169-73.

17. Huang P, Shi Y, Wang X, Zhang SS, Zhang C. Use of microperimetry to compare macular light sensitivity in eyes with openangle and angle-closure glaucoma. Jpn J Ophthalmol. 2012; $56: 138-44$.

18. Wong EN, Morgan WH, Chen FK. Intersession test-retest variability of 10-2 MAIA microperimetry in fixation-threatening glaucoma. Clin Ophthalmol. 2017;11:745-52.

19. Kameda T, Tanabe T, Hangai M, Ojima T, Aikawa H, Yoshimura N. Fixation behavior in advanced stage glaucoma assessed by the MicroPerimeter MP-1. Jpn J Ophthalmol. 2009;53:580-7.

20. Lima VC, Prata TS, De Moraes CG, Kim J, Seiple W, Rosen RB, et al. A comparison between microperimetry and standard achromatic perimetry of the central visual field in eyes with glaucomatous paracentral visual-field defects. Br J Ophthalmol. 2010;94:64-7.

21. Okada K, Watanabe W, Koike I, Tsumamoto Y, Mishima HK. Alternative method of evaluating visual field deterioration in very advanced glaucomatous eye by microperimetry. Jpn J Ophthalmol. 2003;47:178-81.

22. Orzalesi N, Miglior S, Lonati C, Rosetti L. Microperimetry of localized retinal nerve fiber layer defects. Vision Res. 1998; 38:763-71

23. Sato S, Hirooka K, Baba T, Tenkumo K, Nitta E, Shiraga F. Correlation between the ganglion cell-inner plexiform layer thickness measured with cirrus HD-OCT and macular visual field sensitivity measured with microperimetry. Invest Ophthalmol Vis Sci. 2013;54:3046-51.

24. Tanito M, Nitta K, Katai M, Kitaoka Y, Yokoyama Y, Omodaka $\mathrm{K}$, et al. Differentiation of glaucomatous optic discs with different appearances using optic disc topography parameters: the Glaucoma Stereo Analysis Study. PLoS ONE. 2017;12:e0169858. 